\title{
Erratum to: FAM98A is a novel substrate of PRMT1 required for tumor cell migration, invasion and colony formation
}

\author{
Khondker Ayesha Akter $^{1} \cdot$ Mohammed A. Mansour $^{1,2} \cdot$ Toshinori Hyodo $^{1} \cdot$ Satoko Ito $^{1}$. \\ Michinari Hamaguchi ${ }^{1} \cdot$ Takeshi Senga $^{1}$
}

Published online: 29 January 2016

(C) International Society of Oncology and BioMarkers (ISOBM) 2016

Erratum to: Tumor Biol.

DOI 10.1007/s13277-015-4310-5

The original article contain an error.

The author missed to include the second affiliation for this published online paper.

The corrected author group is shown above and its corresponding affiliation is shown below.

The online version of the original article can be found at http://dx.doi.org/ 10.1007/s13277-015-4310-5.

\footnotetext{
Takeshi Senga

tsenga@med.nagoya-u.ac.jp

1 Division of Cancer Biology, Graduate School of Medicine, Nagoya University, 65 Tsurumai, Showa, Nagoya 466-8550, Japan

2 Biochemistry Section, Department of Chemistry, Faculty of Science, Tanta University, Tanta 31527, Egypt
} 